\title{
How Liquid Based Microbiology Can Change the Workflow in the Microbiology Laboratories
}

\author{
Carla Fontana $^{1,2 *}$, Marco Favaro ${ }^{1}$, Cartesio Favalli ${ }^{1,2}$ \\ ${ }^{1}$ Department of Experimental Medicine and Surgery, "Tor Vergata” University of Rome, Rome, Italy \\ ${ }^{2}$ Clinical Microbiology Laboratories, Foundation Polyclinic Tor Vergata, Rome, Italy \\ Email: ${ }^{*}$ carla.fontana@uniroma2.it
}

Received August 9, 2013; revised September 9, 2013; accepted September 19, 2013

Copyright (C) 2013 Carla Fontana et al. This is an open access article distributed under the Creative Commons Attribution License, which permits unrestricted use, distribution, and reproduction in any medium, provided the original work is properly cited.

\begin{abstract}
Liquid-based microbiology (LBM) is the future for the technological development of microbiology laboratories. In particular, the Eswab system (by Copan) simplifies and streamlines specimen collection and represents the only liquid system supporting the recovery of all types of bacteria (aerobic, anaerobic, and fastidious bacteria). In addition, LBM offers advantages in the efficiency of microorganism recovery and ease of sampling, transport, and storage. LBM also allows the introduction of true automation in the laboratory: either by using Copan ${ }^{\mathbb{B}}$ (Walk-Away Specimen Processor) or any other commercially available specimen processor that utilizes LBM. In this paper, we illustrate how LBM can positively change laboratory workflow by illustrating several years of our experience with LBM. LBM allows clinical specimen optimization and has several important advantages: cost reduction (due to the smaller number of different devices used), time savings for medical or nursing staff (less confusion in collection device selection and fewer samples being collected), time savings for laboratory staff (fewer samples to access and handle for individual investigations), and patient comfort improvement (multiple sample collection can be avoided). A unique collection device for several investigations also guarantees quality due to the uniformity of the sample and standardization of procedures.
\end{abstract}

Keywords: Workflow; Liquid-Based Microbiology; Specimen Processor; Molecular Assays; Rational Use of Laboratory Resources

\section{Introduction}

Automated specimen processors, such as the Walk-Away Specimen Processor (WASP; Copan Italia; Brescia, Italy) and the liquid-based microbiology (LBM) concept have been introduced to microbiology laboratories with the advent of the flocked swabs (FLOQSwabs ${ }^{\mathrm{TM}}$ by Copan). Flocked swabs, composed of many thousands of perpendicularly sprayed-on short nylon fibers to the tip of an applicator, collect more sample volume than traditional swabs (which, on the contrary, are traditional fiber wrapped swabs that act like an absorbent mattress, entrapping a large proportion of the sample within the fibers). The fibers of flocked swabs when in contact with liquid surface automatically and completely release the sample. The ESwab system (a flocked nylon swab transported in $1 \mathrm{ml}$ of liquid Amies transport medium) is one of the most representative of the flocked swab family [1,2]. Eswab and WASP were introduced by Copan Italia in 2005 and 2008, respectively. Since the development of

${ }^{*}$ Corresponding author. the ESwab tube collection system, many other devices have been developed. Today, there are no clinical specimens in any branch of the microbiology laboratory that cannot be processed on the WASP. The increased efficiency of LBM has been extensively demonstrated $[3,4]$. The advantages of LBM combined with the WASP are the standardization of the seeding process and preservation of the viability of microorganisms, including those that are particularly difficult to culture [1-5]. However, in an era reduced funding for laboratories and with evidence that many laboratories are experiencing growing shortages of trained microbiology technologists and technicians, there is considerable interest in automation that could potentially lessen labor demands for specimen processing [5]. In our laboratory, we adopted the LBM system in 2008 and used LBM for a variety of tests, including Gram staining, culturing, and molecular assays. This wide use of the LBM system allows us to reduce and rationalize the use of the different device used for specimen collection (e.g., those destined for culturing and several different devices for molecular biology as- 
says). We use "one swab for all", i.e., for the majority of microbiology investigations. Once collected and delivered to the laboratory, the LBM device enters a laboratory workflow with the WASP in the middle and several branches that reach out to different areas of the laboratory. In this paper, we describe our experience of four years by using the LBM system and automation to demonstrate how radically but usefully this system changes laboratory workflow.

\section{Materials and Methods}

In this study, we have used ESwab, Fecal-swab, SL-solution, LIM broth, and BHI broth (Copan Italia; Brescia, Italy). ESwab is used for the microbiological specimens usually collected with a swab, such as genital, nasal, oropharyngeal, ocular, ear, and wound specimens. They are used for traditional culturing (in seeding automation using the WASP system), Gram stain smear preparation, direct detection of bacterial antigens and toxins, and molecular assays. Fecal swab (FS) is a system designed to collect, transport and preserve fecal specimens. Particularly, FS medium is a modified formula of Cary Blair broth specifically intended to support viability of enterobacteria. SL-solution is a ready to use system for liquefying sputum specimens (it contains dithiothreitol as active principle). SL-solution is used to pre-treat mucus-rich respiratory specimens for Gram smears, culturing, and molecular tests. LIM broth (a tube containing 2 $\mathrm{ml}$ of LIM broth and a regular flocked swab used for the collection, transport, and enrichment of recto-vaginal specimens) was used to detect group B Streptococcus (GBS). LIM broth is a modification of Todd Hewitt broth in which the presence of antibiotics inhibits growth of normal bacterial flora and guarantee isolation and preservation of GBS. Finally, BHI broth (a system containing $2 \mathrm{ml}$ of BHI broth, which is ideal to preserve and maintain the viability of microorganisms) was used to store respiratory specimens and Streptococcus pneumoniae strains until the culturing and molecular assays.

\subsection{Processing Urogenital Specimens}

The E-swab tubes were run on the WASP for traditional culturing and used to prepare slides for Gram stain examinations (approximately $50 \mu \mathrm{l}$ were sufficient for culture and $30 \mu \mathrm{l}$ were used for smear preparation; see the following section). For traditional culture, four-streak seeding was performed by the WASP on at least five different media: Columbia CNA (in aerobic and anaerobic conditions), chocolate agar with Isovitalex (under microaerobic conditions), MacConkey agar, and Saboraud dextrose agar (bioMérieux, Marcy l'Etoile, France). After WASP processing, the tubes were transferred to the molecular biology laboratory for molecular assays.

\section{Molecular Assays for Urogenital Specimens}

Samples in Amies medium (contained in the Eswab system) were used to detect pathogens using the following PCR assays: BD ProbeTec ${ }^{\mathrm{TM}}$ ET Chlamydia trachomatis and Neisseria gonorrhoeae amplified DNA assays on the ProbeTec ET ${ }^{\text {TM }}$ System; bacteria and protozoa causing vaginosis (Gardnerella vaginalis and Trichomonas vaginalis) with Affirm ${ }^{\mathrm{TM}}$ VPIII (Becton Dickinson, NJ, USA); and Duplica real-time PCR for Mycoplasma genitalium and Ureaplasma urealyticum (EuroClone, Pero Italy).

In January 2013, some of our molecular assays were switched to a newly introduced system: Anyplex ${ }^{\mathrm{TM}}$ II STI-7 Detection (V1.1) (Seegene Inc., Seoul, Korea), which operates on a CFX96 ${ }^{\mathrm{TM}}$ real-time PCR system (Bio-Rad). The system simultaneously detects $C$. trachomatis, N. gonorrhoeae, M. genitalium, M. hominis, $U$. urealyticum, U. parvum, and $T$. vaginalis from urine, urethral, vaginal, and cervical specimens. To detect $G$. vaginalis, the $G$. vaginalis/Lactobacillus species Real TM Quantitative system (Sacace) was introduced. This system also operates on the CFX96 ${ }^{\mathrm{TM}}$ real-time PCR system (Bio-Rad).

In particular, for C. trachomatis and $N$. gonorrhoeae detection, $10 \mu \mathrm{l}$ of Amies from an Eswab tube were chilled in the sample diluent (Becton Dickinson) and then processed as recommended by the manufacturer. For G. vaginalis and T. vaginalis, $100 \mu \mathrm{l}$ of Amies from an Eswab tube were directly used from the reagent pack of Affirm ${ }^{\mathrm{TM}}$ VPIII according to the manufacturer's instructions. Duplica real-time PCR for M. genitalium and $U$. urealyticum was performed on $100 \mu$ l of Amies according to the manufacturer's instructions. For the newly introduced Anyplex STI-7 and G. vaginalis/Lactobacillus species Real TM Quantitative system, $190 \mu$ l of Amies added to $10 \mu \mathrm{l}$ of the kit internal control was processed according to the manufacturer's instructions.

\subsection{Processing Respiratory Specimens}

Respiratory samples [bronchoalveolar lavage (BAL), bronchial aspirate, (BAS) and sputum (SP)] represent a significant proportion of routine microbiological specimens and are very important for the management of critically ill patients. Copan developed the SL-Solution (SL), a ready-to-use mucus-dissolving solution in tubes with $1.0 \mathrm{ml}$ aliquots. Microscopic examinations were performed after centrifugation $(4000 \times \mathrm{g}$ for $20 \mathrm{~min})$. The slides were prepared using approximately $30 \mu \mathrm{l}$ of the pellet, air dried, fixed at $42^{\circ} \mathrm{C}$, and stained using PREVI COLOR V1 as described above. The validation was performed according to the Murray Washington scheme. The microbial count (expressed in UFC/ml) was performed using the HB\&L system (Alifax, Padova Italy) and took account of the dilution ratio made by SL [6]. 
The SL tube was sent to the WASP for seeding on a number of selective growth media and selective differential media (Columbia CNA, chocolate agar, MacConkey agar, Saboraud agar, or Schaedler agar), incubated at $37^{\circ} \mathrm{C}$ under aerobic, microaerobic, and anaerobic conditions, and observed for five days.

\section{Molecular Assays on Respiratory Specimens}

One milliliter of BAL, BAS, and SP were added to a SL tube to obtain a $1: 1$ ratio (sample/SL, with the exception of samples tested on GeneXpert, for which a ratio of 2:1 is recommended by the manufacturer), vortexed, and used immediately. Smears of SL-treated samples were prepared immediately. Nucleic acid was extracted from all samples $(500 \mu \mathrm{l}$ of SL solution/sample) with the EZ1 Robot (Qiagen Inc.). The DNA obtained from all samples was tested for the agents listed below [7].

DNA extracted from a mix of specimens/SL solution was used to detect respiratory pathogens. In particular, Duplicate real-time Chlamidophila pneumoniae, Mycoplasma pneumoniae, and Legionella pneumophila (EuroClone S.p.A.; Pero, Italy) (which we named RESP) run on the Smart Cycler platform were used to detect $C$. pneumoniae, M. pneumoniae and L. pneumophila. Mycobacterium tubercolosis complex (MTB) was detected by using the Xpert ${ }^{\circledR}$ MTB/RIF, while mycobacteria other than $M$. tuberculosis (MOTT) were detected using the Genotype Mycobacteria Direct (Hain Lifescience GmbH, Nehren, Germany).

We have recently introduced new detection systems. Anyplex $^{\mathrm{TM}}$ II RB5 detection (Seegene) is able to detect $C$. pneumoniae, M. pneumoniae, L. pneumophila, Bordetella pertussis, and Bordetella parapertussis simultaneously. The Anyplex ${ }^{\mathrm{TM}}$ MTB/NTM Detection system is a multiplex real-time PCR system for the detection of $M$. tubercolosis and non-tubercolosis mycobacteria and for the detection of isoniazid/rifampicin resistance in the case of MTB-positive results. Both systems are used starting with SL solution and then run on a CFX96 ${ }^{\mathrm{TM}}$ real-time PCR system (Bio-Rad) according to the manufacturer's instructions.

\subsection{Processing Wound Swabs}

Five hundred microliters of Amies from Eswab-wound infections (either from ulcers from diabetic patients or from surgical infection) were pre-enriched in HB\&L vials (Alifax) [6,8]. Briefly, the HB\&L ${ }^{\text {TM }}$ system uses yellow-capped vials for the culture test and blue-capped vials for a residual antimicrobial activity test (RAA test). The RAA test was not performed in this study. The medium present in both vials supports the growth of a wide variety of microorganisms. Because many microorganisms are difficult to culture, the medium was also supplemented with $0.2 \mathrm{ml}$ of difficult element broth (DEB;
Alifax) containing nicotinamide adenine dinucleotide, factor $\mathrm{X}$, and hemin. After a $6 \mathrm{~h}$ of pre-enrichment, the mixed broth/specimens were transferred into sterile tubes for automation (sterile red cupped tubes; Copan) and plated by WASP (five streak seeding was used) onto Columbia CNA, MacConkey agar, or Saboraud agar, incubated at $37^{\circ} \mathrm{C}$ and observed for five days. For microscopic examinations, $30 \mu \mathrm{l}$ of Amies was placed onto the surface of a slide, spread, fixed, and stained as described above. The microscopic examinations were evaluated following the Q score method of Matkosky et al. [9].

\section{Wound Specimens and Molecular Tests}

Two hundred microliters of the broth from HB\&L preenriched vial (previously inoculated with Amies from ESwab-wound) were used to extract DNA using EZ1 (Qiagen Inc., Valencia, CA), and $1 \mu \mathrm{l}$ of the eluate was used in the amplification reaction for the 16S rDNA gene (primers $8 \mathrm{~F}$ and $516 \mathrm{R}$ ). Three microliters of the amplicon was used for a sequencing reaction using the BigDye Terminator v1.1 Cycle Sequencing-ready reaction kit (ABI PRISM) (Applera, Foster City, CA, USA). The electropherograms were run on the ABI 310 and genetically analyzed (Applera); the mixed sequence was analyzed by direct sequencing with Mixed Rip Seq (Isentio, Bergen, Norway) [10].

\subsection{Processing Stool Specimens and Rectal and Perineal Swabs}

Stool specimens (4 - $5 \mathrm{mg}$ ) were collected on a FS in the ward and then delivered to the laboratory. The FS is for gastrointestinal sample collection (stool, rectal swab, and perineal swab). The collection kit consists of a rectal swab and vial containing $2 \mathrm{ml}$ of liquid Cary-Blair transport medium specifically developed for the collection, transport, and viability of enteric microorganisms and nucleic acid stability during transportation at room temperature.

The most common investigation was for Salmonella, Shigella, and Campylobacter (SSC) culture. Upon arrival, the FS was briefly vortexed, and $500 \mu$ of each sample was inoculated in a Copan Selenite Automation tube containing $2 \mathrm{ml}$ of selenite broth and incubated at $37^{\circ} \mathrm{C}$ for $6 \mathrm{~h}$. The samples were then plated onto different selective agars using the WASP. For the investigation of specific pathogens, such as Campylobacter spp., enterohemorrhagic and enteropathogenic Escherichia coli (EHEC and EPEC respectively), $100 \mu \mathrm{l}$ of Cary-Blair was directly used with the micro-immunochromatographic system, such as ImmunoCard STAT! ${ }^{\circledR}$ CAMPY or ImmunoCard STAT! ${ }^{\circledR}$ EHEC (Meridian Bioscience, Inc.; Cincinnati, OH, USA). In the case of positive results, $30 \mu \mathrm{l}$ of Cary-Blair was plated onto the surface of Campylosel selective agar (bioMérieux) using the WASP, 
incubated at $37^{\circ} \mathrm{C}$ under microaerobic conditions, and observed for $72 \mathrm{~h}$ to determine the antimicrobial susceptibilities of the isolates. In the case of a positive result for EHEC and EPEC, $30 \mu \mathrm{l}$ of Cary-Blair from the FS was plated onto $\mathrm{O} 157$ Selective Agar (bioMèrieux). For Yersinia detection, $30 \mu \mathrm{l}$ of Cary-Blair from the FS was plated directly onto the surface of Yersinia CIN agar (bioMérieux), incubated at $37^{\circ} \mathrm{C}$ under aerobic conditions, and observed for $48 \mathrm{~h}$. For these pathogens, the seeding was performed by the WASP.

Clostridium difficile toxins were detected using Xpert $^{\mathrm{TM}}$ C. difficile (Cepheid Europe, Vira Solelh, France) by pipetting $150 \mu \mathrm{l}$ of Cary-Blair from the FS into the sample reagent and then following the manufacturer's instructions. For the culture of $C$. difficile, $30 \mu \mathrm{l}$ of Cary-Blair was plated directly onto the surface of Chro$\mathrm{mID}^{\mathrm{TM}}$ (bioMèrieux) and incubated under anaerobic conditions until sufficient growth for the investigation of antimicrobial susceptibilities.

For the rectal and perineal swabs, the specimens were collected by using Eswab. Five hundred microliters of Amies was transferred to a tube of Selenite broth (Copan). After $6 \mathrm{~h}$ of selective enrichment, the tubes were directly sent to the WASP for seeding onto the selective agar previously described for SSC culture from fecal specimens. Then, $30 \mu \mathrm{l}$ of Selenite broth was plated using the five streak seeding method.

\subsection{Processing Recto-Vaginal Specimens for GBS}

LIM broth was used to collect the recto-vaginal specimens for GBS investigation. LIM broth was incubated for $16-24 \mathrm{~h}$ at $37^{\circ} \mathrm{C}$, and at the end of the selective enrichment period, $30 \mu \mathrm{l}$ was streaked onto the surface of Brilliance GBS (Oxoid) by the WASP, incubated at $37^{\circ} \mathrm{C}$ under aerobic conditions and observed at 24 and $48 \mathrm{~h}$ [11-13].

\subsection{Other Specimens}

We also recently introduced perineal swabs for the screening of intestinal carriers of Acinetobacter baumannii-multidrug resistant (ABAU) and Carbapenem-resistant Enterobacteriaceae (CRE) in our hospital. Both use Eswab as the collection system. Thirty microliters of Amies is streaked onto specific chromogenic media (CHROMAGAR ACINETOBACTER (pbi international; Milan, Italy) and Brilliance CRE Agar (Oxoid Ltd. Cambridge; UK)) by the WASP. The plates are incubated at $37^{\circ} \mathrm{C}$ under aerobic conditions and observed for $48 \mathrm{~h}$ $[14,15]$.

\subsection{BHI Broth}

This is a new device from Copan that contains $2 \mathrm{ml}$ of
BHI. This product is used in our laboratory to preserve the viability of microorganisms in specimens for which culture is not possible at the time of delivery (for example, during the night) or preserve the viability of pathogens to delay some molecular assays (for example, the molecular genotyping of S. pneumoniae (data not shown).

\subsection{Gram Stain of the Specimens}

Slides prepared using $30 \mu \mathrm{l}$ of Amies were air dried or gently warmed at $42^{\circ} \mathrm{C}$ and then stained using PREVI COLOR V1 Gram Stainer (bioMérieux) according to the manufacturer's instructions [3].

\section{Results}

Our experience with and extensive use of LBM devices demonstrates that a laboratory operating in manual-mode can automate several processes by changing specimen collection from traditional systems of collection (including those destined to molecular assays) to liquid phase methods (Figure 1). The same specimen collected with LBM devices can be used for smear preparation destined for Gram stain, culture, antigen/toxin detection, and numerous other molecular assays. This change does not affect the sensitivity but instead improves the workflow by reducing processing time similar to direct sequencing.

We examined an average of 200 of urogenital specimens per week. Of these, $80 \%$ combined traditional culture investigations with molecular assays (such as those for the detection of sexually transmitted infections: $C$. tracomatis, N. gonorrhoeae, G. vaginalis, T. vaginalis, Mycoplasma, and Ureaplasma). Particularly for the

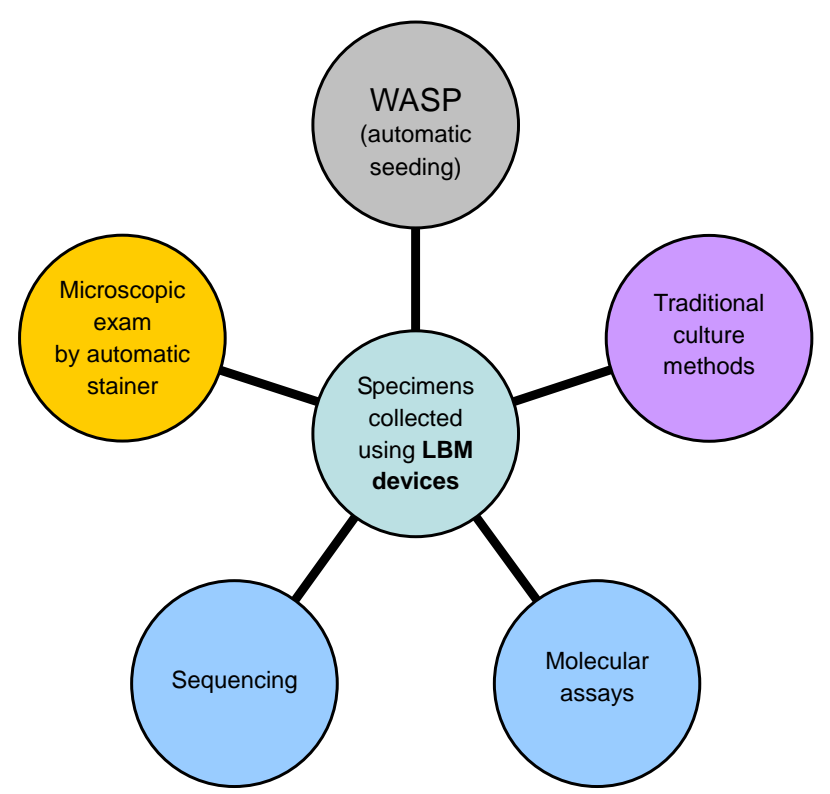

Figure 1. Central role of LBM devices in a multidirectional and multi-tasking laboratory. 
pathogens listed above, the use of Eswab did not reduce the percentage of positive samples expected from previous determinations, i.e., $1.5 \%$ for $\mathrm{CT}, 1.3 \%$ for $\mathrm{NG}$, $13.78 \%$ for $\mathrm{GV}$, less than $0.2 \%$ for $\mathrm{TV}$, and approximately $10 \%$ for Mycoplasma [16]. The culture results were also excellent, as has been reported in previous studies $[4,5,17,18]$.

Considering the results for respiratory specimens, we have routinely processed 7052 samples (3461 BAL, 947 BAS, and 3713 SPU) to date, of which approximately $70 \%$ combined traditional culture investigations with molecular assays (MOTT, MTB, and RESP). The SL solution is excellent for recovering microorganisms by culture on the WASP and does not interfere with molecular assays. It is user friendly and enables rapid and uniform fluidization without pre-incubation. The only criticism regards microscopic examinations (particularly Gram staining); to preserve the integrity of cellular as well as microbial details, the smear must be prepared immediately after the dissolving of respiratory specimens in SL solution. There is not a significant inhibition of the amplification reactions of the various molecular assays. In fact, it was necessary to re-analyze only $10 \%$ of the samples processed, and the majority of these were sputum, which are especially difficult to treat because they are rich in mucus.

Very interesting comparative results were obtained using Mixed RipSeq (Isentio) versus culture in the wound swabs. Our preliminary findings from 15 wound swabs (from a total of 5956 wound swabs examined by Eswab) showed that the mixed sequencing performed on the Liquid Amies from ESwab after enrichment on HB\&L medium enabled us to detect some pathogens lost in culture. In particular, in five wound swabs, a mixed culture was identified that included Gram negative $(P$. mirabilis, A. baumannii, and B. melitensis), Gram-positive (S. aureus), and anaerobic bacteria (Prevotella disiens) that were lost with the traditional culture.

We have processed over 5000 specimens, 1500 rectal swabs, and 100 perineal swabs. The FS has been shown to be a good device for transporting and storing gastrointestinal pathogens. It has been used alone, for antigen/toxin pathogen investigations, and combined with selective broth, particularly with Selenite broth, by WASP automation. In these circumstances, the recovery and detection components and the products of the bacteria did not present any negative effects. Compared with receiving stools in dry containers, the percentage of positive specimens remained substantially the same for all pathogens, with the exception of $C$. difficile and EHEC. During this time, we isolated and identified a case of EHEC (O113 positive for the $v t x 1$ gene; this strain was kindly characterized by Dr. Caprioli from ISS RomeItaly (data not shown)). In Germany, the WHO reported an alert for cases of hemolytic uremic syndrome (HUS) and EHEC (available at the following web site http:// www.who.int/csr/don/2011_06_02/en/index.html). This was our first and only case of EHEC. For C. difficile, the prevalence of the isolates after the introduction of the FS (routinely began mid-2010) changed from 14\% (87 C. difficile - positive stool specimens in 625 specimens examined in 2010) to $19 \%$ (115 positive in 607 specimens in 2011) and then 26\% (164 positive stools in 633 specimens examined in 2012). However, the high prevalence in 2012 could be explained by a local spread of a cluster of C. difficile NAP1/027 (43 isolates) not previously observed. The molecular assays on fecal samples have also shown good results, with the only exception being a few specimens in which the C. difficile toxins were present in amounts near the detection threshold of the Xpert ${ }^{\mathrm{TM}} C$. difficile assay (particularly because they were collected in patients previously treated for C. difficile infections). Therefore, beginning the test from the FS could cause an increase in $\mathrm{CT}$ such that a borderline positive sample could be a negative result [19].

LIM broth for the detection of GBS has advantages in the recovery of this pathogen, and our data confirm the findings previously described. The prevalence of the isolates observed in 2009, when the FS was not routinely used, compared with the present are significantly increased: $2.9 \%$ vs. $7.6 \%$, respectively [17].

Finally, the results from screening intestinal carriers of ABAU and CRE are also promising, even if the number of specimens tested is too low to correctly evaluate the prevalence of such isolates in colonized patients.

\section{Discussion}

Since the development of the ESwab tube collection system, many other products have been developed. Thus, today there are no or very few clinical specimens in each branch of the microbiology laboratory that cannot be processed on the WASP and that have been improved by the introduction of LBM devices $[1,2,4,11,17,18]$.

In our laboratory, we adopted the LBM system in 2008 and used it for a variety of tests, including culture, Gram staining, and many molecular assays. WASP automation has improved the laboratory workflow by re-allocating staff to specialized sections of the laboratory [20-22]. The Copan LBM device family has allowed us to optimize the workflow in the laboratory, especially for its suitability for a variety of testing methods, such as Gram stain smear preparation and cell culture with manual and automated inoculation methods, and for many molecular assays $[1,3,17,18,22]$. LBM is used for culturing assays and allows clinical specimen optimization and has several important advantages: cost reduction (due to the smaller number of different devices used); time savings for medical or nursing staff (less confusion in collection 
device selection and fewer samples being collected); time savings for laboratory staff (fewer samples to access and handle for individual investigations); and patient comfort improvement (multiple sample collection can be avoided). A unique collection device for several investigations also guarantees quality due to the uniformity of the sample and standardization of procedures. Finally, LBM devices processed by an automated instrument allow a greater standardization linked to requirements of sample and process traceability [5,20-22].

Our findings demonstrate the appreciable changes in the workflow and, of course, the advantages due to the LBM introduction. Unification of collection systems can reduce manual processing and determine the standardization of procedures. All of these are basic stages that microbiologists must prepare to accept and introduce in the laboratory for good microbiological practice and the benefit of the patient. The real challenge for the microbiologists, in the next years, is the ability to catch the novelties and to introduce them in the diagnostic process also by adapting and connecting them to several different technologies. We believe that our work is an example of this process of changing.

\section{Acknowledgements}

We gratefully acknowledge Pelliccioni M., Di Traglia L, Basile E., Magnanti M., Falcione F., Mauti A. for their technical assistance.

\section{REFERENCES}

[1] K. G. Van Horn, C. D. Audette, D. Sebeck and K. A. Tucker, "Comparison of the Copan ESwab System with Two Amies Agar Swab Transport Systems for Maintenance of Microorganism Viability," Journal of Clinical Microbiology, Vol. 46, Suppl. 5, 2008, pp. 1655-1658. http://dx.doi.org/10.1128/JCM.02047-07

[2] K. G. Van Horn, C. D. Audette, K. A. Tucker and D. Sebeck, "Comparison of 3 Swab Transport Systems for Direct Release and Recovery of Aerobic and Anaerobic Bacteria," Diagnostic Microbiology and Infectious Diseases, Vol. 62, No. 4, 2008, pp. 471-473.

http://dx.doi.org/10.1016/i.diagmicrobio.2008.08.004

[3] C. Fontana, M. Favaro, D. Limongi, J. Pivonkova and C. Favalli, "Comparison of the eSwab Collection and Transportation System to an Amies Gel Transystem for Gram Stain of Clinical Specimens," BMC Research Notes, Vol. 2, No. 1, 2009, p. 244. http://dx.doi.org/10.1186/1756-0500-2-244

[4] G. Jones, R. Matthews, R. Cunningham and P. Jenks, "Comparison of Automated Processing of Flocked Swabs with Manual Processing of Fiber Swabs for Detection of Nasal Carriage of Staphylococcus aureus," Journal of Clinical Microbiology, Vol. 49, No. 7, 2011, pp. 27172718. http://dx.doi.org/10.1128/JCM.00504-11

[5] P. P. Bourbeau and B. L. Swartz, "First Evaluation of the
WASP, a New Automated Microbiology Plating Instrument," Journal of Clinical Microbiology, Vol. 47, No. 4, 2009, pp. 1101-1106. http://dx.doi.org/10.1128/JCM.01963-08

[6] C. Fontana, M. Favaro, S. Minelli, M. C. Bossa, A. Altieri and C. Favalli, "A Novel Culturing System for Fluid Samples," Medical Science Monitor, Vol. 15, No. 2, 2009, pp BR55-BR60.

[7] U. Edwards, T. Rogall, H. Blocker M. Emde and E. C. Bottger, "Isolation and Direct Complete Nucleotide Determination of Entire Genes Characterization of a Gene Coding for 16S Ribosomal RNA," Nucleic Acid Research, Vol. 17, No. 19, 1989, pp. 7843-7853.

http://dx.doi.org/10.1093/nar/17.19.7843

[8] M. Schmohl, S. Beckert, T. O. Joos, A. Königsrainer, N. Schneiderhan-Marra and M. W. Löffler, "Superficial Wound Swabbing: A Novel Method of Sampling and Processing Wound Fluid for Subsequent Immunoassay Analysis in Diabetic Foot Ulcerations," Diabetic Care, Vol. 35, No. 11, 2012, pp. 2113-2120.

http://dx.doi.org/10.2337/dc11-2547

[9] C. Matkosky, E. S. Susan and L. K. Deanna, "Evaluation of the Q Score and Q234 System fro Cost Effective and Clinically Relevant Interpretation of Wound Cultures," Journal of Clinical Microbiology, Vol. 44, No. 5, 2006, pp. 1869-1872.

http://dx.doi.org/10.1128/JCM.44.5.1869-1872.2006

[10] O. Kommedal, B. Karlsen and O. Saebø, "Analysis of mixed Sequencing Chromatograms and Its Application in Direct 16S rRNA Gene Sequencing of Polymicrobial Samples," Journal of Clinical Microbiology, Vol. 46, No. 11, 2008, pp. 3766-3771.

http://dx.doi.org/10.1128/JCM.00213-08

[11] J. S. Heelan, J. Struminsky, P. Lauro and C. J. Sung, "Evaluation of a New Selective Enrichment Broth for Detection of Group B Streptococci in Pregnant Women," Journal of Clinical Microbiology, Vol. 43, No. 8, 2005, pp. 896-897.

http://dx.doi.org/10.1128/JCM.43.2.896-897.2005

[12] A. Trotman-Grant, T. Raney and J. D. Bard, "Evaluation of Optimal Storage Temperature, Time, and Transport Medium for Detection of Group B Streptococcus in Strep B Carrot Broth," Journal of Clinical Microbiology, Vol. 50, No. 7, 2012, pp. 2446-2449. http://dx.doi.org/10.1128/JCM.00238-12

[13] B. R. Berg, J. L. Houseman, M. A. Garrasi, D. W. Newton and C. L Young, "Culture-Based Method with Performance Comparable to That of PCR-Based Methods for Detection of Group B Streptococcus in Screening Samples from Pregnant Women," Journal of Clinical Microbiology, Vol. 51, No. 10, 2013, pp. 1253-1255. http://dx.doi.org/10.1128/JCM.02780-12

[14] A. T. Kerri, W. W. L. Hsiao, A. D. Harris, C. C. Stine, D. A. Rasko and J. K. Johnson, "Patients with Acinetobacter baumannii Bloodstream Infections Are Colonized in the Gastrointestinal Tract with Identical Strains," American Journal of Infection Control, Vol. 38, No. 9, 2010, pp. 751-753. http://dx.doi.org/10.1016/j.ajic.2010.03.005

[15] A. Adler, S. Navon-Venezia, J. Moran-Gilad, E. Marcos, D. Schwartz and Y. Carmeli, "Laboratory and Clinical 
Evaluation of Screening Agar Plates for Detection of Carbapenem-Resistant Enterobacteriaceae from Surveillance Rectal Swabs," Journal of Clinical Microbiology, Vol. 49, No. 6, 2012, pp. 2239-2242. http://dx.doi.org/10.1128/JCM.02566-10

[16] M. C. Salfa, V. Regine, M. Giuliani, M. Ferri, B. Suligoi e la Rete Nazionale dei Laboratori per le Infezioni Sessualmente Trasmesse, "La Sorveglianza delle Infezioni Sessualmente Trasmesse Basata su una rete di Laboratori: 16 Mesi di Attività," Notiziario ISS, Vol. 23, No. 10, 2010, pp. 11-15.

[17] N. A. El Aila, I. Tency, G. Claeys, B. Saerens, P. Cools, H. Verstraelen, M. Temmerman, R. Verhelst and M. Vaneechoutte, "Comparison of Different Sampling Techniques and of Different Culture Methods for Detection of Group B Streptococcus Carriage in Pregnant Women," BMC Infectious Diseases, Vol. 10, 2010, p. 285. http://dx.doi.org/10.1186/1471-2334-10-285

[18] C. A. Rivers and J. R. Schwebke, "Viability of Trichomonas vaginalis in Copan Universal Transport Medium and eSwab Transport Medium," Journal of Clinical Mi-

\section{List of Abbreviation}

(LBM) liquid based microbiology,

(WASP) walk away specimens processor,

(GBS) group B Streptococcus,

(BAL) bronchoalveolar lavage,

(BAS) bronchial aspirate,

(SP) sputum,

(MTB) Mycobacterium tubercolosis complex, crobiology, Vol. 46, No. 9, 2008, pp. 3134-3135. http://dx.doi.org/10.1128/JCM.00841-08

[19] C. E. Gyorke, S. Wang, J. L. Leslie, S. H. Cohen, J. V. Solnick and C. R. Polage, "Evaluation of Clostridium difficile Fecal Load and Limit of Detection during a Prospective Comparison of Two Molecular Tests, the Illumigene C. difficile and Xpert C. difficile/Epi Tests," Journal of Clinical Microbiology, Vol. 51, No. 1, 2001, pp. 278-280. http://dx.doi.org/10.1128/JCM.02120-12

[20] P. P. Bourbeau and N. A. Ledeboer, "Automation in Clinical Microbiology” Journal of Clinical Microbiology, Vol. 51, No. 6, 2013, pp. 1658-1665. http://dx.doi.org/10.1128/JCM.00301-13

[21] O. Dumitrescu, O. Dauwalder and G. Lina, "Present and Future Automation in Bacteriology," Clinical Microbiology and Infection, Vol. 17, No. 5, 2011, pp. 649-650. http://dx.doi.org/10.1111/j.1469-0691.2011.03511.x

[22] G. Greub and G. Prod'hom "Automation in Clinical Bacteriology: What System to Choose?" Clinical Microbiology and Infection, Vol. 17, No. 5, 2011, pp. 655-660. http://dx.doi.org/10.1111/j.1469-0691.2011.03513.x

(MOTT) mycobacteria other than M. tuberculosis, (RESP) M. pneumoniae, C. pneumoniae, L. pneumophila, (FS) fecal swab,

(SSC) Salmonella, Shigella, and Campylobacter, (EHEC) enterohemorrhagic E. coli, (EPEC) enteropathogenic E. coli, (ABAU) Acinetobacter baumannii-multidrug resistant, (CRE) Carbapenem-resistant Enterobacteriaceae. 